\title{
Line profile variations in the $\delta$ Scuti star FG Virginis: A high number of axisymmetric modes ${ }^{\star}$
}

\author{
L. Mantegazza and E. Poretti \\ INAF - Osservatorio Astronomico di Brera, Via E. Bianchi 4623807 Merate, Italy \\ Received 26 August 2002 / Accepted 26 September 2002

\begin{abstract}
Five consecutive nights of high resolution spectroscopic observations of the $\delta$ Scuti star FG Vir allowed us to analyze the radial velocity and line profile variations induced by non-radial multi-mode pulsations. Ten modes were detected. All of them have a photometric counterpart (Breger et al. 1998). Preliminary identifications have been performed by means of the simultaneous fit of line-profile and light variations. Most of these modes have an axisymmetric character $(m=0)$, one is retrograde $\left(9.20 \mathrm{~cd}^{-1}, m=1\right)$ and one is prograde with a relatively high order $\left(21.55 \mathrm{~cd}^{-1}, \ell=3, m=-1\right)$. The more accurate results regard the dominant mode $\left(12.72 \mathrm{~cd}^{-1}\right)$ which is axisymmetric with $\ell=1$. In this case we were able to obtain reliable has a rather low inclination (about $15^{\circ}$ ): this is probably the reason for the high number of observed axisymmetric modes. Hence, since the measured $v \sin i$ is $20.7 \mathrm{~km} \mathrm{~s}^{-1}$, we deduce that the rotation velocity should be around $80 \mathrm{~km} \mathrm{~s}^{-1}$ and the rotational period about $1.5 \mathrm{~d}$.
\end{abstract} \\ estimates of its physical characteristics. The analysis of our data tends to support the idea that the stellar rotation axis probably
}

Key words. stars: individual: FG Vir - stars: oscillations - stars: variables: $\delta$ Sct - techniques: spectroscopic

\section{Introduction}

FG Vir is a case study among $\delta$ Scuti stars. Breger et al. (1998) detected 24 independent frequencies by means of the analysis of its lightcurve, obtained in the framework of a multisite photometric campaign carried out in 1995. They also report on the full observational history of the star. Successively, Breger et al. (1999a) analyzed the phase shifts between different colours of those data, thus exploiting the possibility to discuss the identification of the $\ell$ degrees of the modes; they also compared the observed pulsation characteristics with those predicted by theoretical models.

Other pulsation models of this star were computed by Guzik et al. (2000) and Templeton et al. (2001) and close comparisons, not fully satisfactory, with the observed frequencies were made and discussed.

Intermediate-resolution spectroscopy and simultaneous uvby photometry were gathered by Viskum et al. (1998) in 1996. They derived estimates of the $\ell$ degree for 8 modes from the ratios between the amplitudes of the equivalent width and photometric variations. Their results are generally in agreement with those by Breger et al. (1999a), but this is not surprising because Balona (2000b) showed that the two techniques are not independent. Finally, they suggested that a $3.5 \mathrm{~d}$-variability detected in all observables could be the fingerprint of the

Send offprint requests to: L. Mantegazza,

e-mail: luciano@merate.mi . astro.it

* Based on observations collected at ESO-La Silla, Proposal 55.E-0399 stellar rotation. If this phenomenon is real and its interpretation is correct it would imply an inclination of the rotation axis of about $40^{\circ}$.

Despite the interest in this star and the importance of lineprofile variations in the study of nonradial pulsations, only one night of high resolution spectroscopy has been obtained so far on FG Vir. Such a night was simultaneous with the 7 nights of photometry performed by Mantegazza et al. (1994) in 1992. However, owing to the short baseline and the complex pulsation spectrum of the star, only preliminary results were proposed about the mode identification on the basis of these observations. Nevertheless, a low $v \sin i$ value of about $21 \mathrm{~km} \mathrm{~s}^{-1}$ and the indication that the rotation axis should have a relatively low inclination (about $30^{\circ}$ ) were obtained.

Therefore, we decided to complement the photometric campaign by Breger et al. (1998) with a high-resolution spectroscopic survey of FG Vir, as we did for many other $\delta$ Scuti stars (Mantegazza 2000; Mantegazza et al. 2001).

\section{Observations}

The observations were performed during 5 consecutive nights (April 7-12, 1995) with the Coudé Echelle Spectrograph (CES) attached to the $1.4 \mathrm{~m}$ Coudé Auxiliary Telescope (CAT) at the La Silla Observatory (ESO) via Remote Control from Garching. The CES was used with the short camera in the blue path configuration and a resolving power of 60000 was set $(0.075 \AA$ of resolution). The spectrograms cover the range between 4490 and $4526 \AA$ A. 100 spectrograms with exposure 


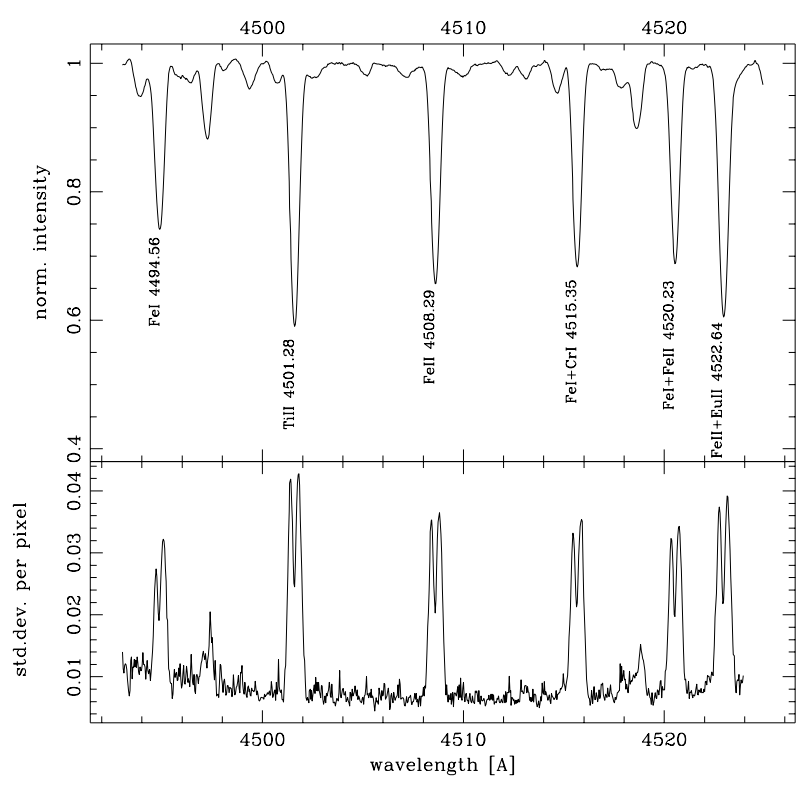

Fig. 1. Upper panel: average spectrum with the identification of the main lines. Lower panel: variance of each pixel about the average spectrum.

times of 15 min were obtained in the 5 nights, covering about 35 hours of observation. The $S / N$ at the continuum level was about 250 in the better observing conditions; however due to the presence of cirrus on some nights the average $S / N$ is about 180 . The spectra were flat fielded, rebinned into wavelengths and extracted in the standard way using the MIDAS package. The resulting ASCII files were then cleaned of cosmics, normalized to the continuum and rebinned to heliocentric wavelengths by means of our own codes. Figure 1 shows the average spectrum with the identifications of the main lines (upper panel) and the standard deviations about the mean of each pixel (lower panel). This panel clearly shows that there are strong variations in the lines profiles. The double peaks of the standard deviation curve indicate that the main contribution to this variation is due to axisymmetric modes $(m=0)$.

\section{Radial velocities}

The radial velocities $(R V)$ were derived by cross-correlating the normalized spectrograms with the average one; in this way we benefit from the information supplied by all the available lines. The resulting time series was then frequency analyzed by means of the successive least-squares power spectrum technique for multiperiodic signals (Vaniceek 1971; Antonello et al. 1986). Due to the limited resolution of our data, at each iteration of our frequency analysis we replaced the frequencies of the deteced modes with the corresponding more accurate photometric values by Breger et al. (1998). This approach allowed us to detect 10 periodic components. Nine of them correspond to the 9 strongest photometric modes; the remaining one $\left(34.119 \mathrm{~cd}^{-1}\right)$ is an isolated high frequency mode (the 13 th photometric term; the 17 th photometric term may also interact).

The $R V$ and their fit according to this solution are reported in Fig. 2. The uncertainty on the amplitudes, as derived by the formula given by Breger et al. (1999b), is of $0.05 \mathrm{~km} \mathrm{~s}^{-1}$.
Table 1. Amplitudes of the 10 components detected from the frequency analysis of the $R V$ curve (second column) and radial velocity amplitudes of the 14 strongest photometric modes (3rd column; see text for discussion).

\begin{tabular}{cccc}
\hline \hline Freq. & \multicolumn{2}{c}{$R V$ amplitude } & $V$ amplitude \\
& 10 terms & 14 terms & \\
$\mathrm{cd}^{-1}$ & \multicolumn{2}{c}{$\left[\mathrm{km} \mathrm{s}^{-1}\right]$} & {$[\mathrm{mmag}]$} \\
\hline 12.716 & 1.39 & 1.39 & 21.1 \\
24.228 & 0.41 & 0.55 & 4.5 \\
23.403 & 0.25 & 0.26 & 4.1 \\
21.052 & 0.44 & 0.46 & 3.7 \\
19.868 & 0.34 & 0.33 & 3.5 \\
12.154 & 0.37 & 0.36 & 3.5 \\
9.656 & 0.19 & 0.18 & 3.4 \\
9.199 & 0.23 & 0.23 & 3.1 \\
19.228 & 0.27 & 0.21 & 1.5 \\
20.288 & - & 0.09 & 1.3 \\
24.200 & - & 0.12 & 1.3 \\
16.074 & - & 0.05 & 1.0 \\
34.119 & 0.14 & 0.14 & 1.0 \\
21.232 & - & 0.13 & 1.0 \\
& & & \\
rms res. & 0.32 & 0.31 & \\
\hline
\end{tabular}

The full amplitude of the $R V$ curve is only $4 \mathrm{~km} \mathrm{~s}^{-1}$, but the residual $\mathrm{rms}$ is an order of magnitude smaller. The detected frequencies (first column) and their $R V$ amplitudes (second column) are reported in Table 1, in order of ranking of the photometric $V$ amplitudes (last column). The $12.716 \mathrm{~cd}^{-1}$ term is still the predominating, as in the photometry. The other terms act as perturbing ones, modifying the cycle-to-cycle shape of the main one, which however remains clearly distinguable in the intranight curves. Note also that the ranking of the $R V$ and light amplitudes are very similar.

In order to check how much these amplitudes can be affected by neglecting other undetected modes, we performed another fit including also the 4 largest photometric modes undetected in the $R V$ curve analysis. The new $R V$ amplitudes are reported in the third column. As we can see, the inclusion of the new terms does not affect appreciably most of the amplitudes, with the exception of $19.228 \mathrm{~cd}^{-1}$ and $24.228 \mathrm{~cd}^{-1}$. This can be easily explained by interaction effects in the least-squares solution: a new term $\left(21.232 \mathrm{~cd}^{-1}\right)$ is a $2 \mathrm{~cd}^{-1}$ alias of the former one and another $\left(24.200 \mathrm{~cd}^{-1}\right)$ is very close to the latter.

Finally we carefully checked the $R V$ variations in order to get some hint of the presence of the $3.5 \mathrm{~d}$ variability detected by Viskum et al. (1999), even if this period does not emerge from the frequency analysis. To do this we compared also the zero-points of the individual nights obtained from the 10 components fit of all the data. The five datapoints have a standard deviation of $0.10 \mathrm{~km} \mathrm{~s}^{-1}$, which is comparable to the mean rms error of the individual datapoints of $0.08 \mathrm{~km} \mathrm{~s}^{-1}$. This quantity puts an upper limit to the amplitude of this variation. No evidence of this variation has be found in the photometric data by Breger et al. (1998; Breger, private communication). 


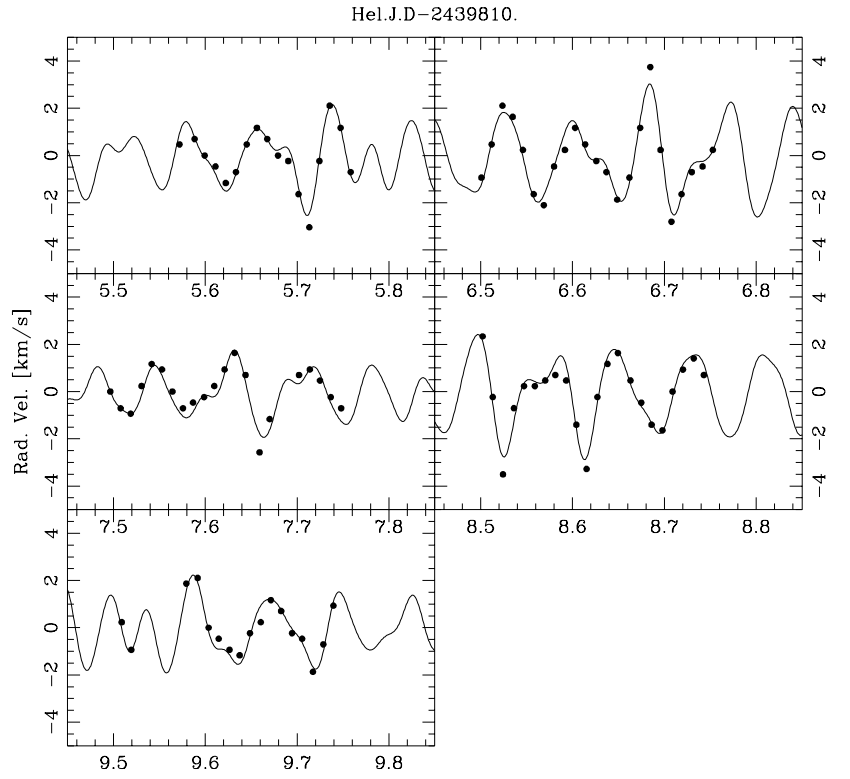

Fig. 2. Radial velocity variations and their least-squares best fit with 10 components.

\section{Analysis of line profile variations}

The analysis of the line profile variations (hereinafter lpv) was performed by means of the pixel-by-pixel analysis. A detailed description of this approach was made by Mantegazza \& Poretti (1999) and by Mantegazza (2000). In the case of FG Vir the lines are rather narrow, covering about 35 pixels. Therefore we tried to get the most accurate profiles as possible. To do that we computed an average profile for each spectrogram by adding the lines at $4501.28,4508.29,4515.34$ and $4520.23 \AA$. The alignment was performed by cross-correlating these features. When superposed, the profiles of these lines are almost exactly coincident and the resulting mean profiles are characterized by an average $S / N$ of about 350 . We didn't use two other lines at 4494.56 and $4522.64 \AA$ because they were slightly wider, when superposing their profiles on to those of the others. This can be explained by the fact that the two lines are actually a blend or, more likely, by the fact that they lie close to the blue and red borders of the spectra, respectively, and there the local continuum is not well defined (see Fig. 1).

Once more, we used the photometric values to select the correct value among the highest peak and its $\pm 1 \mathrm{~cd}^{-1}$ aliases.

We were able to identify 10 terms: the 9 strongest photometric ones (already detected in the $R V$ curve) and the term at $21.55 \mathrm{~cd}^{-1}$, which ranks 18 th (with a $V$ amplitude of $0.8 \mathrm{mmag}$ ) among the photometric modes, while in the lpv analysis it is the 4 th. We can also see that this term has been not detected in the $R V$ curve. These simple facts already suggest it is a non-radial mode with a relatively high $\ell$ degree.

The detected frequencies are listed, in order of detection, in Table 2 together with the percent variance reduction and the mean amplitude (expressed as percent of the local continuum amplitude) across the whole line profile, $S / N$ (in amplitudes) in the pixel where each mode has its maximum amplitude and finally the mean $S / N$ across the whole line profile. It is evident
Table 2. Components detected from the frequency analysis pixel by pixel of the variations of the average line profile in order of detection with their percent variance reduction, average amplitude across the whole line profile, $S / N$ in the pixel with maximum amplitude and mean $S / N$ across the whole line profile.

\begin{tabular}{rccrr}
\hline \hline $\begin{array}{r}\text { Freq. } \\
{\left[\mathrm{cd}^{-1}\right]}\end{array}$ & $\begin{array}{c}\text { Var. Red. } \\
\%\end{array}$ & $\begin{array}{c}<\text { Ampl. }> \\
\text { \% cont. }\end{array}$ & $S / N$ Max & $\langle S / N\rangle$ \\
\hline 12.716 & 7.02 & 2.18 & 42.1 & 22.0 \\
21.052 & 0.35 & 0.49 & 8.7 & 4.3 \\
19.868 & 0.56 & 0.58 & 12.0 & 5.3 \\
21.551 & 0.37 & 0.50 & 10.5 & 4.2 \\
9.199 & 0.33 & 0.51 & 8.8 & 4.0 \\
12.154 & 0.43 & 0.52 & 10.2 & 4.7 \\
19.228 & 0.27 & 0.43 & 7.8 & 3.7 \\
23.403 & 0.33 & 0.48 & 7.9 & 4.0 \\
24.228 & 0.35 & 0.51 & 8.3 & 4.2 \\
9.656 & 0.14 & 0.31 & 5.5 & 2.7 \\
\hline
\end{tabular}

that the $12.72 \mathrm{~cd}^{-1}$ term is by far the strongest, also in the line profile variations.

The behaviours of amplitudes and phases for each mode across the line profile are shown in the panels of Fig. 3. The error bars correspond to the formal errors derived from the least-squares fit. The wavelengths of the line profiles have been transformed into Doppler velocities assuming zero velocity at the line center. It is immediately evident that the dominant mode $\left(12.72 \mathrm{~cd}^{-1}\right)$ has an axisymmetric character having zero amplitude at the line center and having at the same time a rotation of phase of $180^{\circ}$. The same identification also applies to the modes at 12.15 and $21.05 \mathrm{~cd}^{-1}$. The curves of the other modes have more complex behaviours and need a more careful analysis.

\section{Fit of the line profile variations}

The lpv were modeled with different non-radial spheroidal modes in order to find the best fitting ones. The adopted technique is that described by Mantegazza et al. (2001) and Mantegazza (2000); it fits simultaneously lpv and light variations computing for each tested mode $(\ell, m)$ and inclination the parameters that supply the least discriminant (i.e. the squared difference between observed and computed profile and light curve). To model light variations, we have adopted the $v$ amplitudes and phases derived from the multi-site campaign by Breger et al. (1998), whose baseline includes that of our spectroscopic observations. All the modes with $0 \leq \ell \leq 3$ were tested for fixed values of the inclination angle, which in turn was changed from $5^{\circ}$ (the critical value at which the star reaches the break-up velocity is about $3^{\circ}$ ) to $90^{\circ}$.

Despite the small amplitudes of the observed variations (see Table 2) and the short time baseline, we were able to obtain results only partially ambiguous. In particular, we fully exploited the possibility to identify the $m$ orders. To assign $\ell$ numbers was a more difficult task, because the discriminants are more sensitive to the $m$ orders, rather than to the $\ell$ degrees (see also Mantegazza et al. 2001). 

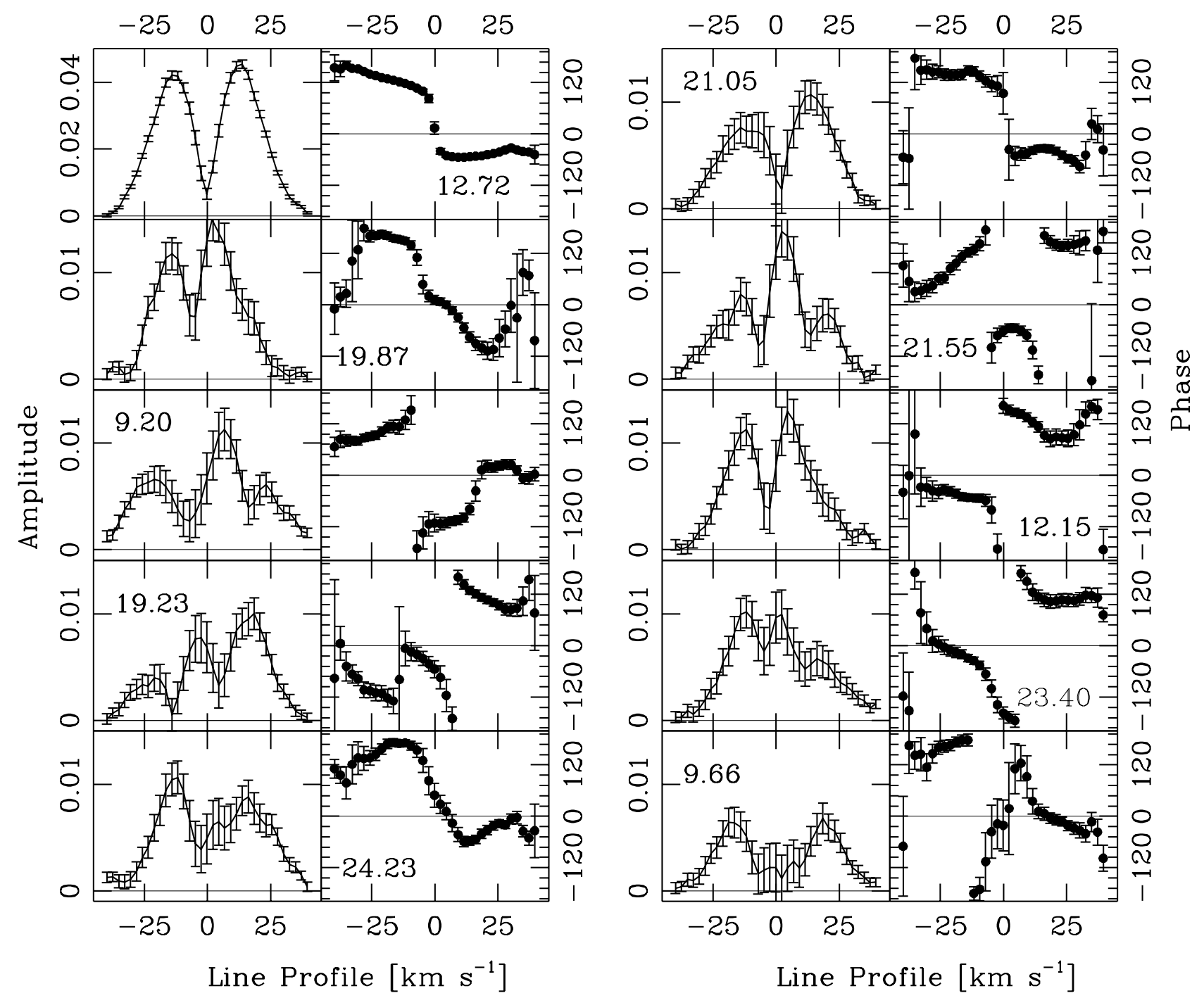

Fig. 3. Behaviour of amplitudes (left panel) and phases (right panels) of the 10 terms detected in lpv. Amplitudes are expressed taking the continuum intensity as unity and phases in degrees. Wavelengths have been transformed into Doppler velocities with respect to the centre of the line.

\subsection{Inclination of the rotation axis}

The discriminants of the $12.72 \mathrm{~cd}^{-1}$ mode, whose $1 \mathrm{pv}$ are by far the best defined, strongly support its identification as $\ell=1$, $m=0$, and at the same time constrain the inclination of rotation axis to be less than about $40^{\circ}$. In order to obtain stronger constraints we computed a global discriminant as a function of inclination. To do that we selected for each fixed inclination and for each detected mode the discriminant corresponding to the identification $(\ell, m)$ which gives its best fit. The global discriminant is the sum of these individual ones at each fixed inclination.

The result is shown in Fig. 4 (solid line): we see that this function suggests $i<35^{\circ}$ with a most probable value between $10^{\circ}$ and $15^{\circ}$.

Another approach to estimate this value is to profit from the photometric identification of the $\ell$ values performed by Breger et al. (1999a). A new total discriminant has been computed at each fixed inclination by summing the individual least discriminants of the best fitting modes corresponding to these $\ell$ degrees. We used the five modes for which Breger et al. (1999a, Table 2) supply an unambiguous identification (12.72, $21.05,19.87,12.15$ and $\left.9.20 \mathrm{~cd}^{-1}\right)$. The result is shown as a dotted line in Fig. 3. We see that again it favours low inclinations with a most probable value around $15^{\circ}$.

\subsection{Mode identification}

Regarding the mode identification, we have already said that the $12.72 \mathrm{~cd}^{-1}$ results quite unambiguously $\ell=1, m=0$. Assuming a low inclination for the rotation axis $\left(\sim 15^{\circ}\right)$, we get the result reported in the second and third columns of Table 3. In the two successive columns we compare the $\ell$ values derived by us to those obtained from photometry (Breger et al. 1999a), and from intermediate-resolution spectroscopy (Viskum et al. 1998). We can see our $\ell$ values show uncertainties of about \pm 1 .

From the $m$ values we can see that most of the modes are axisymmetric $(9.20,12.15,12.72,19.2319 .87,21.0523 .40$ and 


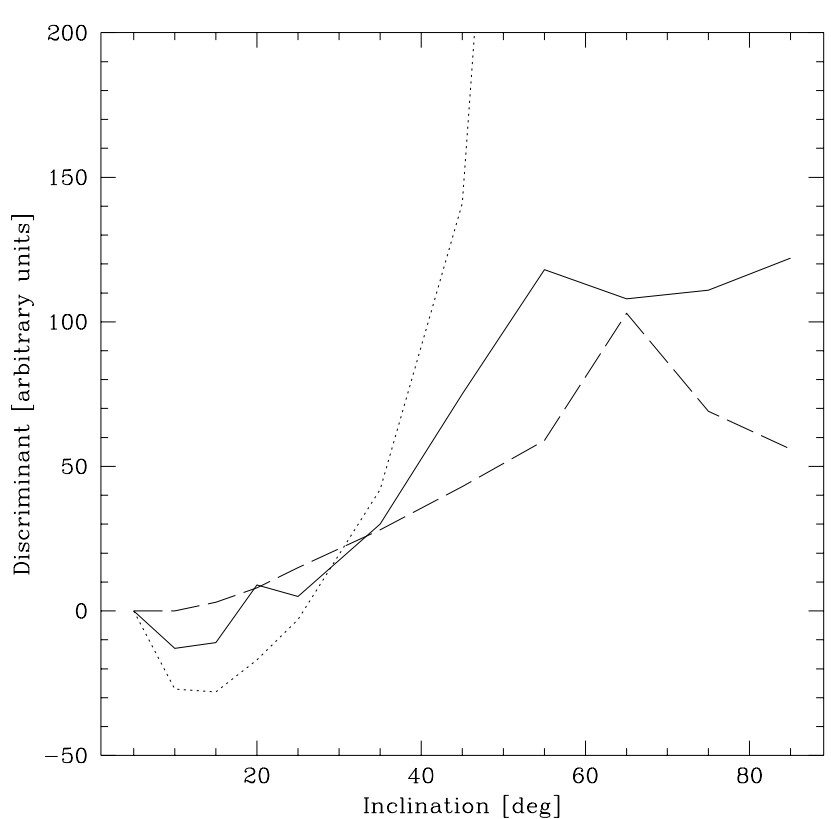

Fig. 4. Behaviour vs. inclination of rotation axis of various discriminants. Solid line: sum of the least discriminants at a given inclination for all the detected modes; dotted line: sum of the discriminants of the 5 modes with unambiguous $\ell$ values supplied by Breger et al. (1999a); long dashed line: discriminant of the $21.55 \mathrm{~cd}^{-1}$ mode, the only one of relatively high degree, and therefore without photometric identification.

Table 3. Identifications of the main modes detected from line profile variations.

\begin{tabular}{ccccc}
\hline \hline Freq. & $\begin{array}{c}\text { lpv } \\
\text { Present }\end{array}$ & $\begin{array}{c}\text { Phot. } \\
\text { Breger et al. } \\
(1999 \mathrm{a})\end{array}$ & $\begin{array}{c}\text { Spect. } \\
\text { Viskum et al. } \\
(1998)\end{array}$ \\
$\mathrm{cd}^{-1}$ & $\ell$ & $m$ & $\ell$ & $\ell$ \\
\hline 9.20 & 1,2 & 1 & 2 & 2 \\
9.66 & 0,1 & 0 & 1,2 & 2 \\
12.15 & $2,1,0$ & 0 & 0 & 0 \\
12.72 & 1 & 0 & 1 & 1 \\
19.23 & 0,1 & 0 & - & - \\
19.87 & $2,1,0$ & 0 & 2 & 2 \\
21.05 & 1,0 & 0 & 2 & 2 \\
21.55 & 3 & -1 & - & - \\
23.40 & $1,0,2$ & 0 & 0,1 & 0 \\
24.23 & 0,1 & 0 & 1,2 & 0 \\
\hline
\end{tabular}

$24.23)$, one is prograde with $m=-1\left(21.55 \mathrm{~cd}^{-1}\right)$ and one is retrograde $\left(9.20 \mathrm{~cd}^{-1}\right)$.

The presence of retrograde modes in $\delta$ Scuti stars, even if they are much less frequent than prograde ones, is not uncommon; for instance other cases were found by Mantegazza et al. (2000) in X Cae, by Mantegazza et al. (2001) in BV Cir and by Kennelly et al. (1998) in $\tau$ Peg.

The lpv fitting of the $21.55 \mathrm{~cd}^{-1}$ mode confirms what was said in the previous section, i.e. that it has a relatively high $\ell$ value. The behaviour of the discriminant of the best fitting mode $(3,-1)$ as a function of the inclination supports the hypothesis of a low $i$ value, indeed it mimics the shape of the total discriminant (Fig. 4, long-dashed lines).

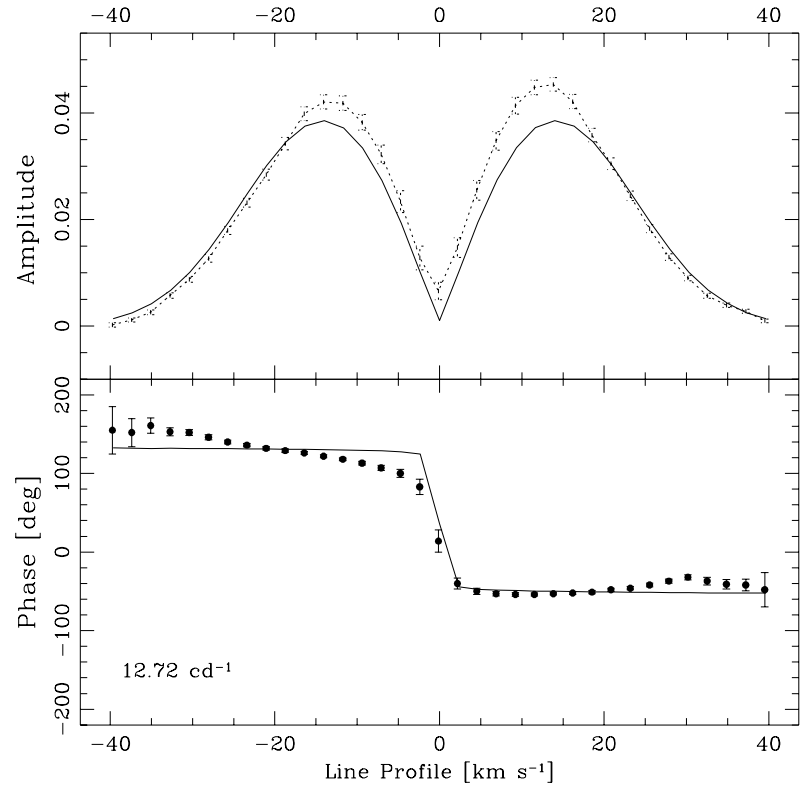

Fig. 5. Behaviour of amplitude (dotted line) and phase (dots) of the $12.72 \mathrm{~cd}^{-1}$ term across the line profile compared with the analogous curves predicted by the best lpv fitting model $\left(\ell=1, m=0, i=15^{\circ}\right.$; solid lines).

The presence of a large number of axisymmetric modes is confirmed by the analysis of the variations of the second moment. It is well known that axisymmetric modes do not show variations in the even moments. In our case, from the frequency analysis of the second moment timeseries we found unambiguously only two terms: 9.20 and $21.55 \mathrm{~cd}^{-1}$, the only two identified by the analysis of $1 p v$ as non-axisymmetric.

A careful inspection of Table 3 can give us a hint about the richness of the pulsational content of FG Vir. All the observed modes have a low $\ell$ value: this is supported not only by our analysis, but also by the independent analyses performed by Breger et al. (1999a) and Viskum et al. (1998). In addition, our analysis strongly suggests that most of the observed modes are axysimmetric. This fact is not a methodological bias, but is based on observational evidence favoured by the small inclination of the rotational axis with respect to our line of sight. We count 9 modes having $m=0$ and all these modes have $\ell \leq 3$. Therefore, we get observational evidence of the suspicion expressed by Breger (2000): we are forced to conclude that hundreds of modes are excited in FG Vir. Moreover, we can be confident that observational techniques are able to measure the effects produced by modes characterized by the same $\ell, m$ couple, but originating from a different $n$ number. Once more, the asteroseismologic interest of $\delta$ Sct stars is confirmed.

\subsection{Rotational velocity}

The fitting procedure of lpv allowed us also to get an accurate estimate of the projected rotational velocity, which gave $v \sin i=20.7 \pm 0.5 \mathrm{~km} \mathrm{~s}^{-1}$. By combining this value with the estimated inclination of the rotational axis, the stellar equatorial rotational velocity is larger than $35 \mathrm{~km} \mathrm{~s}^{-1}$ (just considering $i \leq 40^{\circ}$ ) and possibly about $80 \mathrm{~km} \mathrm{~s}^{-1}$ (considering the best 
Table 4. Physical properties of the $12.72 \mathrm{~cd}^{-1}$ mode with their formal errors.

\begin{tabular}{lrrrrrr}
\hline \hline $\begin{array}{l}\text { Freq. } \\
\mathrm{cd}^{-1}\end{array}$ & $\ell, m$ & $\begin{array}{r}\mathrm{V}_{r} \\
\mathrm{~km} \mathrm{~s}^{-1}\end{array}$ & $\begin{array}{r}\Psi \\
\mathrm{deg}\end{array}$ & $\begin{array}{r}\Delta F / F \\
\%\end{array}$ & $\begin{array}{r}\Delta R / R \\
\%\end{array}$ & $f$ \\
\hline 12.72 & 10 & 2.09 & 107 & 2.15 & 0.147 & 14.6 \\
& & .18 & 6 & .07 & .026 & 2.6 \\
\hline
\end{tabular}

estimate of $i=15^{\circ}$ ). Assuming $R=2.3 R_{\odot}$ (Breger et al. 1999a), the latter value supplies a rotational period of about $1.5 \mathrm{~d}$.

\subsection{Characteristics of the $12.72 \mathrm{~cd}^{-1}$ mode}

The only mode for which we can estimate its physical parameters with confidence is the $12.72 \mathrm{~cd}^{-1}$. Table 4 lists with their formal errors, vertical velocity amplitude, phase difference between flux and and radial variations, percent amplitudes of flux (at the observed wavelength) and radius variations, and their ratio $(f)$. The amplitude variations have been normalized according to the suggestion by Balona (2000a), and they correspond to the amplitudes published in our previous papers (e.g. Mantegazza et al. 2000) divided by $\sqrt{4 \pi}$.

Finally, Fig. 5 compares the behaviour of amplitude and phase of this mode across the line profile as derived from observations with the corresponding curves computed with the best fit with the model $\ell=1, m=0, i=15^{\circ}$. The fit looks satisfactory.

\section{Discussion and conclusions}

The analysis of our spectrograms allowed us to detect 10 terms in the radial velocity curve and 10 in the analysis pixel by pixel of the line profile variations. Only one term is different between the two frequency sets $\left(31.12 \mathrm{~cd}^{-1}\right.$, detected in the $R V$ but not in the lpv, and $21.55 \mathrm{~cd}^{-1}$ vice versa). These terms correspond to modes photometrically identified by Breger et al. (1999a). The model fitting of the lpv with spheroidal modes allowed us to suggest some preliminary identification of these modes: 8 out of 10 modes result to be axisymmetric $(m=0)$ of low degree $(\ell=0,2)$. This is not surprising in view of the fact that our fitting techinque supports a low inclination angle $(i \sim$ $15^{\circ}$ ), which, by a selection effect, should favour the observation of these modes. The presence of such a large number of these modes is not exceptional, because for instance the models by Breger et al. (1999a) predict 17 axisymmetric modes with $\ell \leq 2$ in the range from 9 to $25 \mathrm{~cd}^{-1}$.
One mode is prograde with a relatively high degree $(v=$ $\left.21.55 \mathrm{~cd}^{-1}, \ell=3, m=-1\right)$ and one $\left(v=9.20 \mathrm{~cd}^{-1}\right)$ is retrograde $(\ell=1, m=1)$.

Our inclination value $\left(\sim 15^{\circ}\right)$ conflicts with that proposed by Viskum et al. $\left(1999 ; \sim 40^{\circ}\right)$. This last value is based on the supposed long term variation of $3.5 \mathrm{~d}$ for which there is no evidence in our data. However, according to Viskum et al. (1999), this variation could not be always detectable, therefore the question of its reality remains open.

An accurate estimate of the inclination angle is necessary to derive the equatorial rotational velocity, which in turn is indispensable (see e.g. Goupil et al. 2000) in order to perform meaningful comparisons of observed frequencies with those supplied by theoretical models. It will be therefore of the utmost importance that the next observations will be focused to supply an unambiguous value of this observable.

Due to the complexity shown by the variability of this star and the several excited modes with comparable amplitudes, we believe that these results could be improved and more conclusive answers obtained only by means of a multisite spectroscopic campaign with an adequate baseline.

\section{References}

Antonello, E., Mantegazza, L., \& Poretti, E. 1986, A\&A

Balona, L. A. 2000a, in Delta Scuti and Related Stars, ed. M. Breger, \& M. H. Montgomery, ASP Conf. Ser., 210, 170

Balona, L. A. 2000b, MNRAS, 319, 606

Breger, M. 2000, in Delta Scuti and Related Stars, ed. M. Breger, \& M. H. Montgomery, ASP Conf. Ser., 210, 3

Breger, M., Zima, W., Handler, G., et al. 1998, A\&A, 331, 271

Breger, M., Pamyatnykh, A. A., Pikall, H., \& Garrido, R. 1999a, A\&A, 341, 151

Breger, M., Handler, G., Garrido, R., et al. 1999b, A\&A, 349, 225

Goupil, M. J., Dziembowski, W. A., Pamyatnykh, A. A., \& Talon, S. 2000, in Delta Scuti and Related Stars, ed. M. Breger, \& M. H. Montgomery, ASP Conf. Ser., 210, 267

Guzik, J. A., Bradley, P. A., \& Templeton, M. R. 2000, in Delta Scuti and Related Stars, ed. M. Breger, \& M. H. Montgomery, ASP Conf. Ser., 210, 247

Kennelly, E. J., Brown, T. M., Kotak, R., et al. 1998, ApJ, 495, 440

Mantegazza, L. 2000, in Delta Scuti and Related Stars, ed. M. Breger, \& M. H. Montgomery, ASP Conf. Ser., 210, 138

Mantegazza, L., \& Poretti, E. 1999, A\&A, 348, 139

Mantegazza, L., Poretti, E., \& Bossi, M. 1994, A\&A, 287, 95

Mantegazza, L., Poretti, E., \& Zerbi, F. M. 2001, A\&A, 366, 547

Mantegazza, L., Zerbi, F. M., \& Sacchi, A. 2000, A\&A, 354, 112

Templeton, M., Basu, S., \& Demarque, P. 2001, ApJ, 563, 999

Vanicek, P. 1971, ApSS, 12, 10

Viskum, M., Kjeldsen, H., Bedding., T. R., et al. 1998, A\&A, 335, 549 